\title{
Performance Assessment of Advanced Biological Wastewater Treatment Plants Using Artificial Neural Networks
}

\author{
Harun Türkmenler*\$, Murat Pala** \\ *Department of Environmental Engineering, Faculty of Engineering, Adıyaman University, Adıyaman, Turkey \\ **Department of Civil Engineering, Faculty of Engineering, Adıyaman University, Adıyaman, Turkey \\ (hturkmenler@adiyaman.edu.tr,pala@adiyaman.edu.tr)
}

\begin{abstract}
$\$$ Corresponding Author; Harun Türkmenler, Department of Environmental Engineering, Adıyaman University, Adıyaman, Turkey, Tel: +90 416223 3800, Fax: +90 416223 3809, hturkmenler@adiyaman.edu.tr
\end{abstract}

Received: 29.06.2017 Accepted: 22.09.2017

\begin{abstract}
In this study, the application of Artificial Neural Network (ANN) techniques was used to predict the performance of wastewater treatment plant. The ANN-based model for prediction of effluent biological oxygen demand (BOD) concentrations was formed using a three-layered feed forward ANN, which used a back propagation learning algorithm. Based on the mean absolute percentage error (MAPE), the sum of the squares error (SSE), the absolute fraction of variance $\left(\mathrm{R}^{2}\right)$, the root-meansquare (RMS), the coefficient of variation in percent (cov) values, and ANN models predicted effluent BOD concentration. The $\mathrm{R}^{2}$ values were found to be $94.13 \%$ and $93.18 \%$ for the training and test sets of treatment plant process, respectively. It was found that the ANN model could be employed successfully in estimating the daily BOD in the effluent of wastewater biological treatment plants.
\end{abstract}

Keywords Artificial neural network, Biological oxygen demand, Modeling, Performance assessment, Wastewater treatment plant

\section{Introduction}

Biological Oxygen Demand (BOD), Chemical Oxygen Demand (COD) and Suspended Solid (SS) parameters, design and operating of the treatment systems, checking of whether wastewater discharge limits are suitable for the receiving environment are important parameters used to evaluate the performance of the treatment systems. That the number of treatment plants and the importance of are increasing day by day makes it necessary to apply for new methods in first prediction then in analysis of pollutant parameters.

That the influent parameters of wastewater treatment plants show great changes makes it difficult to run these plants with an optimum performance. Several tools have been tried to be developed to run the plants at optimum performances especially for the last 10 to 15 years. Among these tools, the most commonly used one is a mathematically developed active sludge models, employing differential equations in a form of matrices. Although active sludge models have been being developed through IAWQ
(International Association of Water Quality) since 1987, these models still have some weaknesses and disadvantages. Thus, as an alternative to these developed mathematical models, artificial intelligence technics which are based on neural networks and have more common usage area have been lately started to be used and successful results have been achieved [1].

Traditional modeling techniques used in bioprocesses are based on balance equations together with rate equations for microbial growth, substratum consumption and formation of products. Also, since microbial reactions coupled with environmental interactions are non-linear, time variable and a complex nature [2] of traditional deterministic and empirical modeling have shown some limitations [3]. Moreover, predicting the plant operational parameters using conventional experimental techniques is time consuming and is an obstacle in the way of efficient and effective control of such processes. The ANN-based models were found to provide an efficient and a powerful tool to predict WWTP performance [4]. 
However, in the studies mentioned above, ANN models were configurated as single or multiple input and single (or two) output(s). For instance, Wen et al. predicted BOD [5], Choi et al. modeled Total Nitrogen (TN) [6], Mjalli et al. grouped the input-output data in two vectors (one input and one output) for the first approach and four vectors (three inputs and one output) for the second approach [7]. Most of the studies so far have aimed the prediction of overall treatment plant performance or performance of a particular process, but none of these have considered the consecutive subsections (primary and secondary treatment units) in the whole WWTP. Models considering the main treatment units separately and estimating multiple parameters have not been sufficiently developed yet. It is stated by Mingzhi et al. that there is still no all-inclusive procedure or method to design such intelligent controllers by far because of its semiempirical nature in spite of some successful practical applications [8]. So, studies carried out so far are mostly based on two or three parameters input and single output.

The ANN model is very effective in representing the relationships between input and output variables in nonlinear and complex systems. Moreover, ANN models have been widely applied to address problems in process forecasting and control in water and wastewater treatment $[9,10]$.

In this study, the most critical operation parameters most commonly used in declaration of the pollution level of the wastewater and checking of the performance of the wastewater treatment have been selected as influent-effluent control parameters of the model. These parameters are BOD, COD and SS, TN, Total Phosphorus (TP) and the flow rate. ANN model was developed for the prediction of effluent BOD. The model was performed to the influent and final effluent streams in the Atakoy Advanced Biological Wastewater Treatment Plant (AABWWTP).

\section{Material and Methods}

\subsection{General Description of Advanced Biological Wastewater Treatment Plant}

The objective of the plant is to collect the wastewater from Bakırkoy, Bahcelievler and Bagcilar districts completely and Kücükcekmece and Gaziosmanpasa districts partially, which are currently discharged to Ayamama and Tavukcu Rivers, and therefore polluting the Marmara Sea, through collectors, to convey such wastewater to the AABWWTP, where the wastewater is treated in advanced level, and finally, to discharge the treated wastewater to the environment harmlessly. The treatment plant treats the wastewater produced by a population of 1600000 people, by removing carbon, nitrogen and phosphor with a capacity of $400000 \mathrm{~m}^{3} /$ day. Fig. 1 . shows the process flow diagram of AABWWTP. The sludge produced by the plant is made environmentally harmless in the driers.

Data was taken from the database of AABWWTP of Istanbul/Turkey a period of 306 daily records of the year 2012. This period was satisfactory as it covers all probable seasonal variations in the studied variables. To construct the model structure, totally 6 critical wastewater quality parameters were selected as input variables. The output of the ANN model includes BOD. Total 306 data are used and from this 276 for training and 30 for testing.

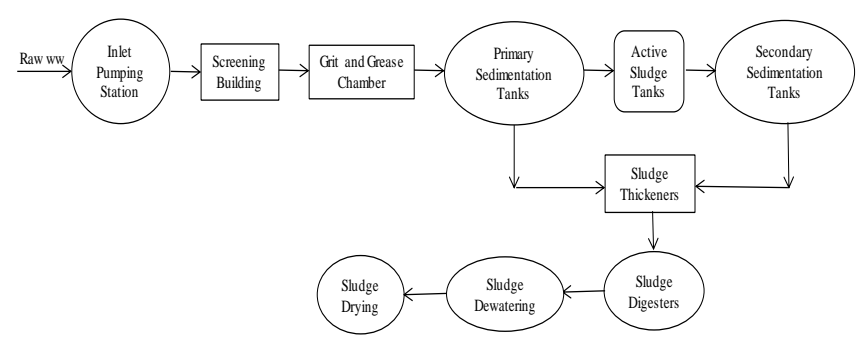

Fig. 1. Process flow diagram of AABWWTP.

\subsection{ANNs}

The ANNs are computer systems developed to automatically fulfil the aim of producing new knowledge by learning and skills like exploring which are exclusive to human brain. It is either extremely difficult or impossible to achieve these skills with traditional computer methods. Therefore, It can be said for artificial neural network to be a computer science branch concentrating on adaptive data processing for cases for which programming is either impossible or extremely difficult [11].

Mjalli et al. has successfully made the predictions of key parameters like BOD, COD and SS values in the effluent in an urban wastewater treatment plant [7]. Cinar monitored the performance of a domestic wastewater treatment plant in terms of BOD, SS and fecal coliform parameters and successfully predicted the effluent parameters with an ANN model [12]. By using a 10-month BOD and SS data of a domestic wastewater treatment plant, Hamed et al. showed that performance could be predicted correctly based on these parameters after a few stages of training [4].

When the interactions between the inputs and outputs of a target events that a neural network is wanted to learn are non-linear, Multilayer Perceptron (MLP) Model can be utilized. Since the pollution level coming into the treatment plant isn't constant and the correlation between parameters can't be stated through equations, the multilayer perceptron model has been used [11].

Economical and easy operation of these wastewater treatment plants at present is an important subject through the utilization of the data that is obtained as the plant is operated in the prediction of the data that will be obtained in the future. It has today been possible to control the plants through modelling in computers by the prediction of operation parameters numerically in advance. ANNs are a data processing system that has a parallel scattering and is composed of many processing units and connections. The ability of ANNs to learn and make generalizations over the relations of the experimental inputs and outputs without a need of any assumption or suggestion provides a big contribution to the studies in this field. 
ANN model was developed to predict effluent BOD concentration for the AABWWTP. ANN is an information processing system inspired by the way such as biological nervous systems e.g. brain. The aim of a neural network is to compute output values from input values via some internal calculations [13].

There are many various kinds of training algorithms. Back Propagation Algorithm (BPA) is one of the most common classes of training algorithms for Feed Forward Neural Networks (FFNNs) [14].

To solve complex problems in many different fields of application such as pattern recognition, identification, classification, speech, vision, and control systems, ANNs have been employed successfully. Today, ANNs can be trained to solve problems which are difficult for conventional computers or human beings. Moreover, one of the plus points of ANNs, different from the conventional approach, is that it extracts the desired information directly from the data. A neuron is the fundamental processing element of a neural network. A biological neuron is based on input from other sources and perform in a way that a general non-linear process on the result of combining them and give the final result. An input layer, some hidden layers, and an output layer usually compose the network [15].

A MLP is the most basic and commonly used ANN. This includes at least three or more layers, including an input layer, an output layer, and a number of hidden layers. Each neuron in one layer is connected to the neurons in the next layer, and there are no connections among the units of the same layer. Depending on the problem, the number of neurons in each layer may change (Fig. 2.). The relationship between inputs and outputs can be represented in an easier way using biases network. Generally, a transfer function including algebraic equations may be linear or non-linear [16].

In context of this study, the influent values of the pollution parameters of the wastewater treatment plant have been tried to be taught to the network. Since the network is to make predictions based on the values of the network, Multilayer Perceptron Model has been chosen. In this network, values are supplied to the network on entrance layer, and after they pass through sub-layers, they go to the output layer, where the answer of the network to the input data is served to the world outside.

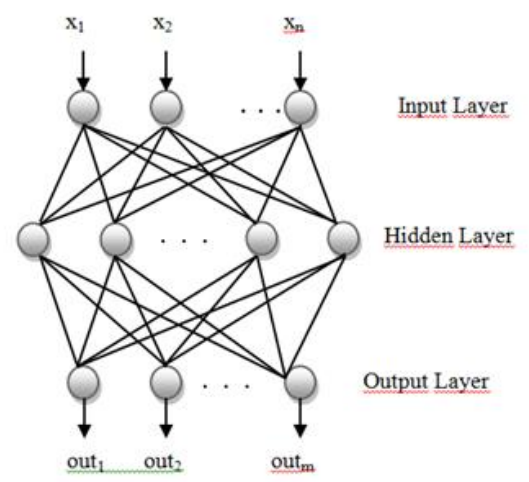

Fig. 2. A typical MLP neural network.
Neural networks are formed by input data vectors, neurons and output functions. Input data to the neuron are transformed by means of a base function and leave by an activation function [17] connection between input and output data and neurons is made by weight factors, which determine the effect of the input variable on the neuron.

The weighted sum of input components is calculated as

$$
\operatorname{Net} j=\sum_{i=1}^{n} w_{i j} x_{i}
$$

where Net $\mathrm{j}$ is the weighted sum of the $\mathrm{j}$ th neuron for the input received from the preceding layer with $n$ neurons, $w_{i j}$ is the weight between the $j$ th neuron and the $i$ th neuron in the preceding layer, and $\mathrm{x}_{\mathrm{i}}$ is the output of the $\mathrm{i}$ th neuron in the preceding layer. The output of the $\mathrm{j}$ th neuron, outj, is calculated using a sigmoid function as follows:

$$
\text { out } j=f\left(\text { Net }_{j}\right)=\frac{1}{1+\exp \left(- \text { kNet }_{j}\right)}
$$

where $k$ is a constant that is used to control the slope of the semi linear region. Non-linear Sigmoid activates in each layer except the input layer [18].

Through a particular training pattern in which it adjusts the weights by a small amount at a time, the BPA, as one of the most famous training algorithms for the MLP, is a gradient descent technique to minimise the error. The conjugate gradient algorithm (CGA) and the scaled conjugate gradient algorithm (SCGA) as several adaptive learning algorithms have newly been explored. In CGA, to determine an appropriate step size, which makes the CGA faster than BPA, a search is made in each iteration along the conjugate gradient direction. SCGA is more effective than BPA and CGA [19]. By using a step size scaling mechanism, SCGA avoids a time-consuming line search per learning iteration [20].

\section{Results and Discussion}

\subsection{ANNs in Certain Formulation of Treatment Process}

In this study, ANN is used to develop a formula based on the logistic sigmoid (logsig) transfer function. The data used for training and testing ANN was obtained from AABWWTP. The result of using the normalization values shown in the Table 1, inputs and outputs are normalized in the (0-1) range. In Table 1, the maximum and minimum values of inputs and outputs are also showed. There are six input parameters in the input layer, namely the $\mathrm{Q}_{\mathrm{w}}$, $\mathrm{BOD}_{\text {influent }}, \mathrm{COD}_{\text {influent }}, \mathrm{SS}_{\text {influent }}, \mathrm{TN}_{\text {influent }}$, and $\mathrm{TP}_{\text {influent }}$ and the output parameter is $\mathrm{BOD}_{\text {effluent }}$ (Fig. 3). The range of inputs and outputs in the trained network is given in Table 1.

With a binary sigmoidal transfer function, the chosen network architecture was 6-9-1 (Fig. 3); SCG was the learning algorithm used. 10000 iterations were performed in order to find out the optimum result. 
Table 1. The range of input and output parameters and normalization values

\begin{tabular}{|c|c|c|}
\hline Parameters & $\begin{array}{l}\text { Range of } \\
\text { values }\end{array}$ & $\begin{array}{c}\text { Normalization } \\
\text { value }\end{array}$ \\
\hline $\mathrm{Q}_{\mathrm{w}}\left(\mathrm{m}^{3} / \mathrm{sn}\right)$ & $5,52-0,99$ & 6,9 \\
\hline $\mathrm{BOD}_{\text {influent }}(\mathrm{mg} / \mathrm{L})$ & $560-120$ & 700 \\
\hline $\mathrm{COD}_{\text {influent }}(\mathrm{mg} / \mathrm{L})$ & $2.352-325$ & 2940 \\
\hline $\mathrm{SS}_{\text {influent }}(\mathrm{mg} / \mathrm{L})$ & $1.744-148$ & 2180 \\
\hline Total-N $\mathrm{N}_{\text {influent }}(\mathrm{mg} / \mathrm{L})$ & $132-26$ & 165 \\
\hline Total-P influent $(\mathrm{mg} / \mathrm{L})$ & $25,50-4,20$ & 31,88 \\
\hline $\mathrm{BOD}_{\text {effluent }}(\mathrm{mg} / \mathrm{L})$ & $37-4$ & 46,25 \\
\hline
\end{tabular}

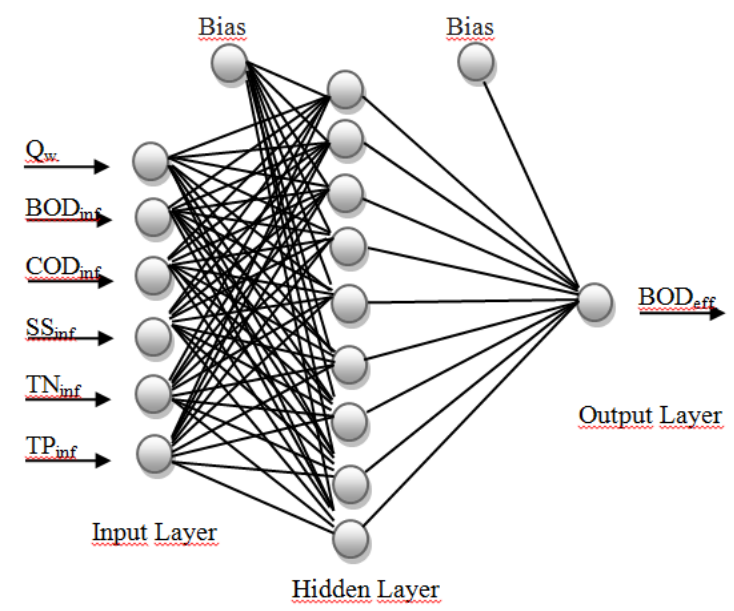

Fig. 3. The architecture of proposed backpropagation neural network.

The network uses the default SCG algorithm for training. The application randomly divides input vectors and target vectors into two sets as follows: $90 \%$ is used for training; $10 \%$ is used to validate that the network is generalizing and to stop training before over-fitting.

For effluent BOD of the training errors are ensured below. Results showed that errors for each condition were quite satisfactory. Thus, the trained Artificial Neural Networks was shown good results quite satisfactory. The performance of training and test sets and the trained ANN statistical parameters are shown in Figures 4 and 5, and in Table 2, respectively. As shown in Figures 4 and 5, and in Table 2, the correlation factor, for any two sets, a pretty high proof of the correctness of trained ANN model.

Table 2. Statistical parameters of the ANN formulation.

\begin{tabular}{lll}
\hline & Training set & Test set \\
\hline MAPE & 23.8079 & 24.3268 \\
SSE & 2.3666 & 0.3334 \\
RMS & 0.0929 & 0.1037 \\
cov & 0.2569 & 0.2743 \\
$\mathrm{R}^{2}$ & 0.9413 & 0.9318 \\
\hline
\end{tabular}

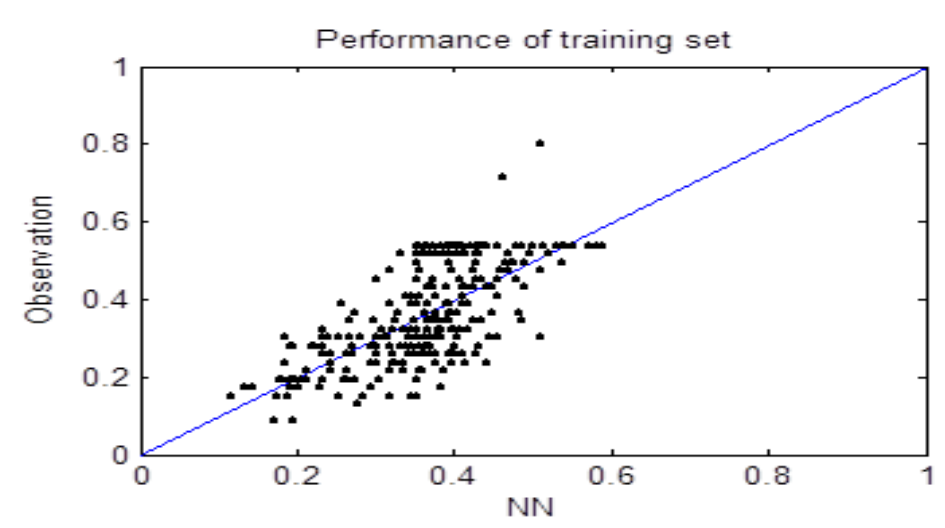

Fig. 4 The performance of the training set.

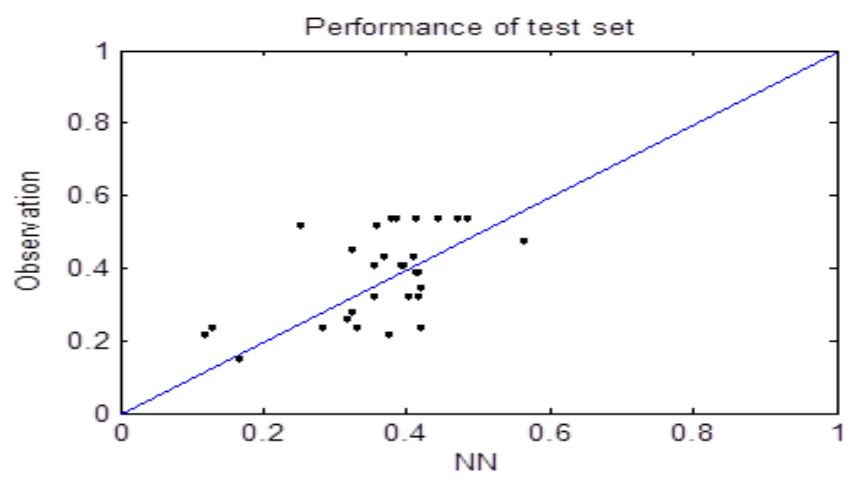

Fig. 5 The performance of the test set.

The MAPE, SSE, $\mathrm{R}^{2}$, RMS, and the cov are defined as follows, respectively:

$$
\begin{aligned}
& \mathrm{MAPE}=\left(\frac{0-t}{0}\right) * 100 \\
& \mathrm{SSE}=\sum_{j}\left(o_{j}-t_{j}\right)^{2} \\
& R^{2}=1-\left(\frac{\sum_{j}\left(t_{j}-o_{j}\right)^{2}}{\sum_{j}\left(o_{j}\right)^{2}}\right) \\
& \mathrm{RMS}=\left((1 / p) \sum_{j}\left|t_{j}-o_{j}\right|^{2}\right)^{1 / 2} \\
& \operatorname{cov}=\frac{\text { RMS }}{o_{\text {mean }}} * 100
\end{aligned}
$$

where $o$ is the output value, $p$ is the pattern, $o_{\text {mean }}$ is the mean value of all output data, and $t$ is the target value.

The maximum MAPEs were found to be $23.8079 \%$ and $24.3268 \%$ and the $\mathrm{R}^{2}$ values to be approximately 0.9413 and 0.9318 for the training and test sets for the effluent BOD model, respectively. In this case, the network response is satisfactory, and simulation can be used for entering new inputs.

ANN models predicted the dynamic behavior of BOD concentrations with good accuracy and provided a very good fit to the training data and the testing data (Figs. 4 and 5). The proposed neural networks can satisfactorily describe the behavior of the process. As a result, the predicted effluent 
BOD rates matched the observed concentrations based on the relatively low SSE, MAPE and very high $\mathrm{R}^{2}$ values, suggesting good prediction performance of the models. When considering the high level of complexity of these biological processes, the broadness of the data range and the computed error values, it is seen that the method successfully predicts the target output.

ANN method is an economical and useful method which can be used in prediction of effluent values and efficiency as the experimental work is both costly and time-consuming. As a consequence of the studies carried out, it has been seen that MLP model, which has been modelled through the data used from the AABWWTP values had a great match with the real data.

\subsection{Certain Formulation of Effluent BOD}

By using the parameters (inputs, weights, normalization factors) of the proposed ANN model, the certain formulation of effluent BOD is derived. From the trained ANN, all necessary parameters are gated, and from the weights of the trained ANN model, the certain expression is formed. In Tables 3 and 4, the weights and bias values in the derivations of ANN based formulations are given. Each input is multiplied by a connection weight.

Table 3. Weight and bias values between input and hidden layers.

\begin{tabular}{cccccccccc}
\hline Weights & \multicolumn{7}{c}{ Number of hidden layer neurons (i) } \\
\cline { 2 - 10 } & 1 & 2 & 3 & 4 & 5 & 6 & 7 & 8 & 9 \\
\hline$w_{1 i}$ & $-22,088$ & $-25,193$ & $-24,737$ & $-12,696$ & 5,5185 & $-8,3588$ & 16,211 & 21,819 & $-76,642$ \\
$w_{2 i}$ & $-13,351$ & $-15,793$ & 21,3465 & $-1,1106$ & $-6,2143$ & 25,6021 & $-12,314$ & $-19,088$ & 25,8532 \\
$w_{3 i}$ & $-1,0371$ & 2,9238 & $-17,403$ & $-1,2963$ & $-6,135$ & $-0,1825$ & $-1,7067$ & 17,7272 & 56,167 \\
$w_{4 i}$ & 1,5861 & 0,59 & $-38,798$ & 4,5001 & $-15,806$ & 18,0896 & 13,325 & 29,2159 & $-20,066$ \\
$w_{5 i}$ & 3,6185 & 5,8039 & 28,1283 & $-18,064$ & 12,3541 & $-25,636$ & $-7,3943$ & $-21,79$ & $-56,363$ \\
$w_{6 i}$ & $-7,3057$ & $-9,3036$ & 16,6211 & 4,632 & 9,5572 & 13,19 & 8,0006 & $-11,485$ & 0,3147 \\
Bias & 19,2864 & 21,6877 & $-6,5005$ & 8,8204 & 5,3474 & $-6,8099$ & $-0,5591$ & 4,0212 & 34,5093 \\
\hline
\end{tabular}

Table 4. Weight values between output and hidden layers.

\begin{tabular}{lccccccccc}
\hline Weights & \multicolumn{10}{c}{ Number of hidden layer neurons (i) } \\
\cline { 2 - 10 } & 1 & 2 & 3 & 4 & 5 & 6 & 7 & 8 & 9 \\
\hline $\mathrm{W}_{\mathrm{i}}$ & 18,8592 & $-16,273$ & 13,5517 & $-4,3086$ & $-9,6423$ & 1,0393 & $-5,6049$ & 16,8257 & 1,0551 \\
\hline
\end{tabular}

In a simple manner, to generate a result first of all products and biases are simply gathered, then transformed through a transfer function (logistic sigmoid), and finally outputs are obtained more easily. Inputs and outputs are normalized prior to the learning process of artificial neural networks. Normalization values to obtain a correct result from the proposed formulas have to be thoroughly considered in this study. Proposed formulation as input parameters obtained from the Table 1 between the maximum and minimum values should be noted that current [21]. 2.8380 is bias value between hidden layer and output.

\section{Conclusions}

In wastewater treatment plants, important parameters of the effluent, BOD, COD, SS, TN and TP, have to be monitored. When these modelling practices are compared to the traditional ones the formers have a lot of advantages. The most important of them is getting the result correctly and quickly. So, by means of using past data for more than one parameter or component predictions can be done toward future and experimental practice need can be minimized and thus, operation costs of the plant can be greatly reduced. Another important advantage of ANNs is that due to their fast-learning ability they can solve a question that they have never encountered before. Therefore, usage of ANNs in modelling practices of wastewater treatment plant has become more common. This paper reveals that estimation of effluent BOD for wastewater treatment process using ANN verifies to be a better technique than conventional mathematical modeling. ANN gives very satisfactory results for the proposed model.

This study confirms the ability of the artificial neural network modeling to forecast the performance of AABWWTP. The proposed certain formulation in the present study will be of help to make this procedure short in a more effective manner than experimental works.

Through the knowledge of the parameters affecting the efficiency of the treatment, new and theoric output and efficiency values can be produced by means of using trained and tested ANN analysis without doing further experimental studies. As a result, ANN is an effective method in prediction of the effluent BOD concentrations of AABWWTP and the efficiency values based on those.

It is concluded that, ANN provides an effective analyzing and diagnosing tool to understand and simulate the non-linear behavior of the treatment process, and is used as a valuable performance assessment tool for plant operators and decision makers. 


\section{Acknowledgements}

The author would like to thank to the Istanbul Water and Sewerage Administration (İSKI), Istanbul, Turkey, for providing the AABWWTP process data used in this study.

\section{References}

[1] Cinar O, Yilmaz A., "Application of Artificial Neural Network Method on Operation of Wastewater Treatment Plant: An Example Study", KSU. Journal of Science and Engineering, 8(2): 48-52, 2011.

[2] Lee D S, Park JM., "Neural network modeling for online estimation of nutrient dynamics in a sequentiallyoperated batch reactor", Journal of Biotechnology, 75: 229-239, 1999.

[3] Cote M, Grandijean B P, Lessard P, Yhibault J., "Dynamic modeling of the activated sludge process: improving prediction using neural networks", Water Research, 29: 995-1004, 1995.

[4] Hamed M, Khalafallah M G, Hassanein E A., "Prediction of wastewater treatment plant performance using artificial neural network", Environmental Modeling and Software, 19: 919-928, 2004.

[5] Wen C. H., Vassiliadis C. A., "Performing hybrid artificial intelligence techniques in wastewater treatment", Engineering Applications of Artificial Intelligence, 11: 685-705, 1998.

[6] Choi D., Park H., "A hybrid Artificial Neural Network As A Software Sensor for Optimal Control of A Wastewater Treatment Process", Water Res., 35: 39593967, 2001.

[7] Mjalli F. S., Al-Asheh S., Alfadala H. E., "Use of artificial neural network black-box modeling for the prediction of wastewater treatment plants performance”, J. Environ. Manage., 83: 329-338, 2007.

[8] Mingzhi H., Ma Y., Jinquan W., Yan W., "Simulation of a paper mill wastewater treatment using a fuzzy neural network", Expert Systems with Applications, 36: 5064-5070, 2009.

[9] Zhang Q., Stanley SJ., "Real-timewater treatment process control with artificial neural network", J Environ Eng. ASCE, 125: 153-160, 1999.

[10] Yu RF., Cheng WP., Chu ML., "On-line monitoring ofwastewater true color using digital image analysis and ANN", J Environ Eng. ASCE, 131: 71-79, 2005.
[11] Oztemel E., "Artificial Neural Networks", Papatya Publication, İstanbul, 2003.

[12] Cinar O., "New tool for evaluation of performance of wastewater treatment plant: Artificial neural network", Process. Biochem., 40: 2980-2984, 2005.

[13] Delgrange V. N., Cabassud N., Cabassud M., DurandBourlier L., Laine J. M., "Neural networks for prediction of ultrafiltration transmembrane pressure: application to drinking water production", Journal of Membrane Science, 150: 111-123, 1998.

[14] Demuth H., Beale M., Hagan M., "Neural Network Toolbox 5: Users Guide", Natick. MA The MathWorks Inc., 2007.

[15] Kalogirou S. A., "Applications of artificial neuralnetworks for energy systems", Applied Energy, 167: 17-35, 2000.

[16] Lu W., "Neural network model for distortional buckling behaviour of coldformed steel compression members", Ph.D. thesis, Helsinki University of Technology, 2000.

[17] Machon I., López H., Rodríguez-Iglesias J., Maran`ón E., Vázquez I., "Simulation of a coke wastewater nitrification process using a feed-forward neuronal net", Environmental Modelling and Software, 22: 1382 1387, 2007.

[18]Pala M., "A new formulation for distortional buckling stress in cold-formed steel members", Journal of Constructional Steel Research, 62: 716-722, 2006.

[19] Berke L., Patnaik S. N., Murthy P. L. N., “Optimum design of aerospace structural components using neural networks", Computers \& Structures, 48(6): 1001-1010, 1993.

[20] Kang H. T., Yoon C. J., "Neural network approaches to aid simple truss design problems", Microcomputers in Civil Engineering, 9: 211-218, 1994.

[21]N., Pala M., Elmas M., Ery1lmaz D. M., “A new approach to determine the base shear of steel frame structures", Journal of Constructional Steel Research, 65: 188-195, 2009. 